\title{
¿Es posible hablar de tolerancia entre iguales? Algunas consideraciones críticas
}

\author{
RENÉ GONZÁLEZ DE LA VEGA \\ Instituto de Investigaciones Jurídicas \\ Universidad Nacional Autónoma de México \\ gonzalezdelavega@yahoo.com
}

\begin{abstract}
Resumen: En la literatura de filosofía moral y política es común que se acepte la tolerancia como una propiedad disposicional, la cual se somete a prueba en repetidas "circunstancias"; una de éstas se refiere a la capacidad o competencia adecuada para poder detener, frenar u obstaculizar el acto objeto de tolerancia. En ese sentido, algunos autores, como Ernesto Garzón, defienden que dicha competencia o capacidad para tolerar no requiere necesariamente la existencia de una relación de supra-subordinación con la persona objeto de tolerancia. No obstante, tras un análisis de lo que se entiende por "competencia" o "capacidad" adecuada y cómo estos términos se articulan con la idea conceptual básica de la tolerancia (pública o privada), se hace una crítica a esta tesis sosteniendo que, para poder hablar de tolerancia, se requiere necesariamente que haya una relación jerárquica de supra-subordinación.

Palabras clave: tolerancia, competencia adecuada, facultades, relaciones de supra-subordinación
\end{abstract}

\begin{abstract}
In the field of moral and political philosophy, it has been unanimously accepted that tolerance is a quality of the agent and it is tested in various circumstances. One of these circumstances refers to the capacity or the adequate competence of the agent to stop, deter or impede the act, which is the object of tolerance. In this context some authors, Ernesto Garzón among them, defend the thesis that such competence or capacity to tolerate does not necessarily require a hierarchical relationship between the tolerating and the tolerated agent. However, in this article the author, after analyzing the meaning of adequate "competence" or "capacity" and its relationship to the basic concept of tolerance (public or private), criticizes the above thesis and argues that tolerance can exist only in hierarchal relationships of supra and subordination.
\end{abstract}

Key words: tolerance, adequate competence, capacity, relationships of supra and subordination

Muchos filósofos de la moral y de la política coinciden en que la tolerancia es una propiedad agencial que puede ser sometida a prueba en reiteradas circunstancias, a las que comúnmente llaman "circunstancias de la tolerancia". Estas "circunstancias" son: (1) la lesión de una convicción relevante; (2) tener el poder o la competencia adecuada para 
frenar, detener o disuadir el acto objeto de tolerancia, y (3) la ponderación de razones a favor de la no intervención. ${ }^{1}$

Las disputas sobre qué "circunstancias de la tolerancia" son suficientes y necesarias para dar cuenta de un concepto "útil" de tolerancia son de sobra conocidas. Pienso, por ejemplo, en el debate suscitado entre filósofos como Annette Schmitt, Andrew J. Cohen y Samuel LaSelva, entre otros, con autores como Ernesto Garzón, Peter Nicholson y D.D. Raphael. Mientras que los primeros consideran suficientes y necesarias las circunstancias que se refieren a: i) la lesión de una convicción relevante, y ii) tener el "poder" o la "competencia adecuada" para intervenir en contra del acto objeto de tolerancia, los segundos consideran necesario agregar al esquema conceptual una tercera circunstancia, concretamente aquella que habla sobre iii) la ponderación de razones a favor de la no intervención.

Como vengo diciendo, el debate sobre qué circunstancias son suficientes y necesarias para hablar propiamente de "tolerancia" es largo e intenso; sin embargo, en este trabajo no es mi intención abordarlo. ${ }^{2}$ Tampoco es mi intención examinar lo que se ha entendido por "convicción lesionada" y "ponderación de los argumentos a favor de la no intervención". Mi propósito, si se quiere, es un poco más modesto. Está únicamente dirigido a analizar algunos de los argumentos que Ernesto Garzón ha introducido a propósito de la segunda circunstancia de la tolerancia y extraer de ellos algunas conclusiones que me parecen relevantes para el tema. ${ }^{3}$

Ahora bien, mi interés en esta circunstancia de la tolerancia surge porque, a diferencia de las circunstancias i) y iii) (la lesión de una

${ }^{1}$ Estas "circunstancias" corresponden a la estructura conceptual de la tolerancia que mayor aceptación ha tenido en la filosofía moral y política contemporánea. Por nombrar algunos de los trabajos más representativos que adoptan esta estructura conceptual, véanse, por ejemplo, Ernesto Garzón Valdés, “'No pongas tus sucias manos sobre Mozart'. Algunas consideraciones sobre el concepto de tolerancia”, el cual se ha incluido en distintas obras; la versión que usaré aquí se encuentra en el libro del mismo autor, Instituciones suicidas. Estudios de ética y política; Annette Schmit, "Las circunstancias de la tolerancia"; Andrew Jason Cohen, "What Toleration Is?"; Rodolfo Vázquez, Liberalismo, Estado de derecho y minorías, y también Entre la libertad y la igualdad. Introducción a la filosofía del derecho, pp. 127-161; Peter Nicholson, "Toleration as a Moral Ideal"; David Daiches Raphael, "The Intolerable"; Richard Vernon y Samuel LaSelva, "Justifying Tolerance"; Thomas M. Scanlon, "The Difficulty of Tolerance"; John Horton, "Toleration as a Virtue"; y la introducción de David Heyd a su libro Toleration. An Elusive Virtue.

${ }^{2}$ He realizado un breve análisis sobre este debate en "Desacuerdos en el concepto de tolerancia".

${ }^{3}$ Hasta donde sé, Ernesto Garzón es quien ha defendido con mayor contunden- 
convicción y la ponderación de razones), ha sido la que menor atención ha recibido en la literatura especializada, aunque al mismo tiempo es la que ha gozado de mayor aceptación. Esta última situación ha generado una especie de descuido teórico que nos puede llevar a pensar que esta "circunstancia" no sufre de la misma clase de problemas que atacan a las otras dos y que se derivan fundamentalmente de sus múltiples interpretaciones. Por lo tanto, no está de más ir avanzando un poco en qué debemos entender cuando se dice que para tolerar, una persona debe de contar con cierta "competencia", "poder" o "superioridad" frente a la persona que cometió el acto objeto de tolerancia. Como decía, en este trabajo abordo estos problemas refutando el argumento según el cual no todos los casos de tolerancia, tanto los que se dan en el ámbito privado como en el ámbito público, implican una relación de suprasubordinación.

Ernesto Garzón usa el pasaje de un cuento de Manuel Vicent publicado en 1983 para ilustrar las bases conceptuales de la tolerancia. En ese pasaje, Vicent describe:

un padre dispuesto a soportar los hábitos de sus hijos, no obstante la aversión que aquéllos provocaban en su sensibilidad de izquierdista moderado con residuos de herencia burguesa. En aras del libre desarrollo de la personalidad y del diálogo abierto, toleró durante años que la alcoba de su hija se [llenara] con una panda de amigos que traían una calaña bastante atroz. No era lo peor que pasaran por delante de sus narices y que no se dignaran saludarle, sino el olor a cabra que dejaban en la sala. Que se limpiaran las botas en la alfombra, que se abatieran sobre las estanterías y manosearan sus libros con las uñas sucias, que se bebieran el whisky y que mearan sin tirar la cadena. Todo esto estaba dispuesto a tolerar. Hasta que, el 14 de mayo de 1980, su hija salió de la leonera con el pelo grasiento y los dedos amarillos de nicotina, cruzó la sala, se dirigió a la biblioteca con la pretensión de llevar a sus compinches la Sinfonía número 40 de Mozart. La tolerancia había llegado a su límite: el padre [...] saltó del sillón impulsado por un muelle y lanzó un grito estentóreo: iiiMozart, no!!!, iiiNo pongas tus sucias manos sobre Mozart!!!

cia la posibilidad de que la tolerancia se dé entre individuos que cuentan con el mismo estatus normativo.

${ }^{4}$ Citado por Ernesto Garzón Valdés en "No pongas tus sucias manos sobre Mozart'. Algunas consideraciones sobre el concepto de tolerancia”, pp. 401-402. Véase Manuel Vicent, No pongas tus sucias manos sobre Mozart. 
La actitud del personaje de Vicent recuerda mucho a la de Mary Warnock cuando dice:

Soy tolerante si uno de los amigos de mis hijas usa sandalias con su traje o bastón con su chaqueta de tweed y no sólo no aludo a este ultraje, sino que hasta expreso mi satisfacción cuando me anuncian su intención de casarse. Practico la virtud de la tolerancia si todavía estoy en buenos términos con mi hijo, a pesar de que cuando está en casa no puedo recoger el desayuno hasta la comida y no estoy segura de que haya quedado whisky en la botella para cuando llame el canciller. ${ }^{5}$

Sin duda, tanto la actitud descrita por Vicent como la descrita por la baronesa Warnock coinciden en varios puntos importantes para el tema de la tolerancia. Sin embargo, el que más interesa resaltar aquí es que ambos personajes se consideran en una posición "jerárquicamente" superior (la de madre o la de padre) que les permite tolerar los actos de sus hijos.

Básicamente, la idea que está detrás de esta circunstancia es la imposibilidad de tolerar algo que se encuentre fuera de nuestra esfera de "competencias". Pero ¿qué debemos entender en este caso por "competencia"? En principio, el término "competencia" nos remite a la idea de reglas regulativas y de derechos. Por ejemplo, siguiendo a Robert Alexy, la idea de "competencia adecuada" es una situación en la que "el status deóntico es opuesto al de sujeción". ${ }^{6}$ Esto es, que el sujeto tolerante está en una situación de superioridad conforme al sujeto cuyo acto tolera o frente al estado de cosas tolerado. ${ }^{7}$ Por lo tanto, no es posible tolerar una tormenta o una ola fuerte de calor, como tampoco lo es tolerar un terremoto. En ciertas circunstancias tampoco decimos que un empleado pueda tolerar a su jefe. De estas cosas se dice que se soportan o que se padecen, pero no que se toleren. Estas situaciones suceden debido a la imposibilidad, fáctica o normativa, de hacer algo en contra de ellas.

Por ello, también suele decirse que, dependiendo de los tipos de competencia que se acepten, puede ampliarse o reducirse el ámbito

${ }^{5}$ M. Warnock, "The Limits of Toleration", p. 125; las traducciones de las citas de esta obra son mías.

${ }^{6}$ R. Alexy, Robert, Teoría de los derechos fundamentales, pp. 234 ss.

${ }^{7}$ Cfr. Javier de Lucas, “¿Para dejar de hablar de la tolerancia?”, p. 120. Como hago notar en el texto, sostengo la posibilidad de tolerar "estados de cosas" siempre y cuando éstos hayan sido generados por seres humanos. Precisamente, debido a la circunstancia ii) de la tolerancia, los estados de cosas generados por fuerzas naturales o fenómenos ajenos a la voluntad humana no entrarían en el ámbito de cosas tolerables (y no tolerables). 
de cosas tolerables (y no tolerables). Si la única clase de competencia que se acepta está basada en la superioridad física o numérica, por ejemplo, está claro que los físicamente débiles o los grupos minoritarios no tendrían ninguna posibilidad de tolerar. De la misma forma, si la única clase de competencia que se acepta es la relacionada con las reglas o con los derechos conferidos por algún sistema jurídico, esto también, aunque en menor medida, reduciría el ámbito de personas con capacidad de tolerar.

Ahora bien, para refutar la idea según la cual todos los casos de tolerancia presuponen una relación de supra-subordinación, Ernesto Garzón introduce una serie de distinciones conceptuales que le sirven para afirmar la posibilidad de una tolerancia entre iguales. Es decir, Ernesto Garzón acepta la posibilidad de que existan actos de tolerancia donde no hay una relación de supra-subordinación entre quien tolera y es tolerado. En No pongas tus sucias manos sobre Mozart, Ernesto Garzón sostiene, como muchos otros filósofos lo hacen, la existencia de una tolerancia pública y otra privada. Especialmente, manteniendo la popular idea de que la tolerancia pública es aquella en que las partes representan grupos de una comunidad política y/o social; y la privada, aquella en la que los participantes son meramente individuos. Es decir, que una es a nivel político o social y la otra a nivel interpersonal.

Por ejemplo, la actitud tolerante de M.A. ${ }^{8}$ hacia el humo que exhala Garzón con cada cigarrillo es un caso típico de tolerancia privada, en contraste con la patente de tolerancia dictada por el emperador José II el 20 de octubre de 1781, en la que estableció la igualdad civil entre católicos y no católicos, la cual se distingue perfectamente como un caso de tolerancia pública.

En este sentido, la Carta sobre la tolerancia de John Locke trata sobre casos típicos de tolerancia pública, los cuentos de Manuel Vicent y Mary Warnock tratan sobre casos de tolerancia privada.

Sin embargo, y a pesar de su ya larga tradición, no hay que apresurarnos en aceptar esta distinción que Garzón abriga con su propuesta. Me parece que, antes de hacerlo, hay que considerar varias cuestiones que la rodean. Dejándome guiar por Manuel Atienza cuando expone algunas de las reglas de aplicación del principio de tolerancia que Garzón desarrolla a lo largo de su obra, veo que Atienza dice que "los límites

${ }^{8}$ E. Garzón, op. cit., p. 184. Este ejemplo es utilizado por Ernesto Garzón en el texto que vengo citando. Las iniciales corresponden al nombre de un colega suyo; por razones de respeto a la intimidad de esta persona he omitido el nombre, a pesar de que Garzón sí lo utiliza en su texto.

Diánoia, vol. LV, no. 64 (mayo 2010). 
para la tolerancia en el plano personal valen, mutatis mutandis, para el plano de la política [o público]".9

Si tomamos en serio la afirmación de Atienza, entonces, ¿por qué no preguntarnos por las diferencias conceptuales que puede haber entre estas dos clases de tolerancia?, ¿o debería aceptarse, sin más, que la única diferencia depende del contexto o del número o de la calidad política de los actores que involucre el acto de tolerancia? Esto ciertamente sería, desde el prisma conceptual, completamente contingente.

Me parece que, para evadir esta clase de críticas y sostener la importancia de esta distinción, Garzón introduce una diferencia más. Esta otra diferencia versa sobre una tolerancia vertical y otra horizontal. Es aquí donde entra en juego lo que hemos llamado la ii) "circunstancia de la tolerancia".

Antes vimos que Garzón, al igual que muchos otros filósofos, acepta como condición necesaria la competencia o el poder para frenar, detener u obstaculizar el acto tolerado. Sin embargo, Garzón también asegura lo siguiente:

la competencia que la tolerancia requiere no debe ser equiparada sin más con una relación de autoridad entre quien tolera y la persona cuyo acto es tolerado [...] tan frecuentes son aquellos [casos] en los que una persona tolera actos de otra con quien se encuentra en una relación de igual jerarquía. ${ }^{10}$

En este tenor, Garzón traza una línea que divide los casos de tolerancia donde la relación de superioridad existe (relación vertical) y los casos en que no existe (relación horizontal). Para defender la existencia de la tolerancia horizontal en el ámbito privado, Garzón pone como ejemplo la aversión al cigarrillo de M.A. que referí antes. Esta relación, en la que puede entrar en juego la tolerancia, se basa, principalmente, "en la creencia — de M.A.- de que el tabaco perjudica su salud"11 y en la necesidad que tiene Garzón de estar rodeado de humo.

Empero, según Garzón, la actitud tolerante de M.A. hacia el humo que él expide entre cada cigarrillo no está fundamentada en una posición jerárquicamente superior a la de él, la cual le permitiría detener o frenar sus hábitos de fumador. En otras palabras, Garzón piensa que

\footnotetext{
${ }^{9}$ M. Atienza, "La filosofía moral de Ernesto Garzón Valdés", p. 25; lo que está entre corchetes es mío.

${ }^{10}$ E. Garzón, op. cit., p. 184.

${ }^{11}$ Ibid., p. 184.
} 
M.A. no se encuentra en una posición jerárquicamente superior a la de él. Por ello, Garzón sostiene que:

Cuando M.A. [...] tolera que fume en su presencia, no lo hace en virtud de alguna posición de autoridad sino, quizá, porque piensa que no es conveniente reducir el círculo de sus interlocutores a quienes han optado por una supuesta salvación de la salud física, o porque considera que no siempre es verdad aquello de mens sana..., o porque considera que también quienes parecen sufrir de una "debilidad terminal de voluntad" [...] pueden, entre cigarrillo y cigarrillo, expresar juicios de algún interés. ${ }^{12}$

A mi juicio, ninguna de las razones esgrimidas por Garzón para justificar el hecho de que M.A. tolere el humo de sus cigarrillos son realmente relevantes frente a la creencia en la que M.A. funda su tendencia a prohibir el acto en cuestión. Pero éste es un problema del que no me voy a ocupar aquí, sobre todo porque tiene que ver con la ponderación entre la convicción lesionada y las razones que se tengan para no intervenir en contra de la acción que la dañó y no con la "competencia" para poder tolerar algo que nos afecta.

Lo que me interesa subrayar con esta cita es que, para Garzón, tanto él como M.A. se encuentran en una posición jerárquicamente igual, pero que, no obstante su horizontalidad jerárquica, M.A. tiene en sus manos la posibilidad de "tolerar" el humo de Garzón. Es decir, que de acuerdo con la definición de tolerancia aceptada por el mismo Garzón, tiene una especie de "competencia" o "superioridad" para hacerlo; competencia que, según el mismo Garzón, deriva de la capacidad que cada cual tiene para defenderse ante daños $u$ ofensas. ${ }^{13}$

Pero, en ese caso, ¿cuál es el criterio que usa Garzón para basar esa "capacidad" que tenemos para defendernos si no es en una especie de "competencia" o de "poder" para hacerlo? A mi juicio, se pueden argumentar varias posibilidades que ayudan a decir por qué M.A. está en una situación de superioridad frente a Garzón. Una primera posibilidad es que de la clase de reclamo (o convicción) que M.A. sostiene se deriva un tipo de "competencia" o "autorización" moral que le permite prohibir, detener o frenar en su presencia los malos hábitos de Garzón. Otra posibilidad es que M.A. se encuentra en ciertas circunstancias o en un contexto que le permite decir que tolera los malos hábitos de Garzón.

Si aceptamos la existencia de esta clase de posibilidades, me parece, entonces, que orillamos a Garzón a tener que elegir entre dos posibi-

${ }^{12}$ Ibid., p. 184.

${ }^{13}$ Ibid., p. 187.

Diánoia, vol. LV, no. 64 (mayo 2010). 
lidades: a) negar la necesidad conceptual de que la tolerancia siempre implique una relación de supra-subordinación (de competencia y sujeción), y, por lo tanto, negar la circunstancia ii) de la tolerancia; o b) aceptar la necesidad conceptual de la segunda circunstancia y, entonces, mantener dos conceptos de "competencia" completamente diferentes: uno donde "competencia" significa libre de sujeción, ${ }^{14}$ y otro donde "competencia" significa una cosa distinta en la que no hay una calidad jerárquica de supra-subordinación.

Lo cierto es que esta segunda forma de entender el término "competencia" es difícil de comprender. Por ejemplo, cuando decimos que alguien tiene la competencia para hacer tal o cual cosa es porque decimos, al mismo tiempo, que está autorizado para hacerlo. Es decir, cuando alguien cuenta con la autorización, la competencia o la capacidad para realizar algo, denota una calidad jerárquica definida frente a los individuos que no la tienen. También es difícil de comprender si aceptamos que existen muy variadas formas de fundamentar y de generar relaciones de supra-subordinación. En el caso de las formas de fundamentación, por ejemplo, éstas en su gran mayoría dependen del sistema normativo en el que esté basada la competencia o autorización, sea que se hable de un sistema moral, de uno jurídico o de uno social. Ello también permite distinguir el ámbito en el que se pueden ejercer distintas facultades: en la iglesia entre curas y feligreses, en la universidad entre profesores y alumnos, en la familia entre padres e hijos, etc. Pero repárese en que todas ellas denotan una calidad jerárquica determinada, la cual depende del sistema normativo del que derivan, y que una persona puede desarrollar en su vida muchos de estos roles: el de padre, el de hijo, el de profesional, el de amigo, el de colega, etcétera.

Ahora bien, la posibilidad de derivar la competencia adecuada de la justificación de nuestras convicciones es una cuita ya aceptada por muchos filósofos morales, entre ellos D.D. Raphael y Peter Nicholson. Por ejemplo, Raphael sostiene que: "[d] esaprobar algo significa que lo juzgas como malo. Un juicio tal, no expresa una preferencia puramente subjetiva, sino que tiene una pretensión de universalidad. Pretende ser aceptado por cualquier agente racional". ${ }^{15}$ Entonces, según Raphael,

\footnotetext{
${ }^{14}$ Esto también se debe a que Ernesto Garzón acepta la misma definición de competencia formulada por Alexy. Garzón dice: "En efecto, la exigencia de competencia para determinar la calidad deóntica del acto permite distinguir entre tolerar y soportar o padecer. Como señala Robert Alexy, lo opuesto a la competencia es la sujeción. El esclavo no tolera los castigos del amo: los soporta o los padece" (Ibid, p. 183).
}

${ }^{15}$ D.D. Raphael, op. cit., p. 139. 
tener un juicio por justificado te da la capacidad para interferir frente a actos de un tercero.

Como dije, una postura muy cercana a la de Raphael es la de P. Nicholson. En el mismo tenor, este último sostiene que "quien tolera reclama que su desaprobación está moralmente justificada y que, por lo tanto, tiene el derecho de actuar en favor de ella: él no sólo tiene el poder, sino la autoridad para hacerlo". ${ }^{16}$ Claro, una cuestión que salta inmediatamente a la vista es que del simple hecho de creer que un reclamo está justificado no se deriva que el reclamo, de hecho, esté justificado. Frente a esto, la postura que adoptan ambos autores es que el reclamo se debe considerar, desde un punto de vista objetivo, moralmente justificado (es por ello que autores como Raphael mencionan la pretensión de universalidad como criterio de corrección del juicio formulado); es decir, que para ellos no bastaría con que el agente crea que su reclamo está justificado para poder decir que cuenta con la "competencia" para tolerar algo, sino que éste tiene que estar objetivamente justificado.

Teniendo en mente todo lo anterior, parecería que el término "competencia" utilizado por los autores que aceptan la ii) circunstancia de la tolerancia, no sólo nos remite al concepto de autoridad, como Peter Nicholson arguye, sino que éste denota otros términos tales como "superioridad", "facultad" o "autorización". También parece que en la mayoría de los casos en los que se habla de "competencia" se hace con referencia a las facultades que nos delegan determinados sistemas normativos, los cuales determinan las facultades que cada individuo tiene en distintas circunstancias.

Por ejemplo, un caso de superioridad o competencia fundamentada en mandatos institucionales puede ser el caso del jefe de una empresa que no permite fumar dentro de sus instalaciones. Según esta perspectiva, el empleado fumador no tiene la competencia necesaria para tolerar o no las reglas de su jefe; si no lo hace, le puede costar el puesto. Ahora imaginemos el caso contrario, que el jefe, a pesar de su declarada aversión al cigarrillo, permitiera fumar en las instalaciones de su empresa. En tal caso se diría que es una persona tolerante.

Pongamos el mismo ejemplo, pero ahora con reglas jurídicas de por medio. Supongamos que la empresa a la que me refiero se trata de una empresa donde se procesa petróleo crudo o donde se almacena dinamita o se trata de una mina. En tal caso, la prohibición de fumar no se podría ver como un acto de intolerancia por parte del jefe contra

${ }^{16}$ P. Nicholson, op. cit., p. 161. 
los empleados fumadores; de hecho, el permitir fumar sería una falta jurídica (y una irresponsabilidad moral) de su parte. Aquí se trata, más bien, de un acto de coherencia con las reglas de un sistema jurídico que prohíbe el uso de cigarrillos en lugares de alto riesgo como las minas, las gasolinerías o las fábricas de productos inflamables.

Antes vimos que el personaje de Vicent se presenta como una persona sumamente tolerante frente a sus hijos, al igual que Mary Warnock. Ambos saben que tienen cierta superioridad moral (la de padre o la de madre) frente a ellos, pues presumen que pueden influir de alguna manera en la rectitud (moral o social) de sus actos. Por ello, se puede hablar de que el personaje de Vicent permite que manoseen sus libros y que se beban su whisky, pues está claro que si no pensara tener esa clase de competencia difícilmente podría decir que él no tolera que su hija lleve a sus compinches la Sinfonía número 40 de Mozart. Lo mismo sucede con Mary Warnock cuando dice que tolera que el novio de su hija use bastón con su chaqueta de tweed.

Me parece sumamente complicado pensar en una situación de tolerancia en la que no se manifieste una situación de superioridad. Según lo que hemos visto, el tolerante siempre se encuentra en una situación de superioridad frente al tolerado. Esto es, la tolerancia implica estar en una posición desde la cual se pueda permitir deliberadamente algo que se desaprueba. Por lo tanto, sólo puede predicarse un acto de tolerancia cuando el sujeto tolerante tiene el "poder" o la "competencia" para prohibir o detener lo que es objeto de tolerancia. De esto se deriva una cuestión que a mi juicio es importante subrayar: si un acto de tolerancia es, por definición, una omisión (es decir, no actuar, por determinadas razones, en contra del acto que lesionó una convicción relevante), y si se acepta que, para que alguien "omita" realizar una acción $x$ es, entre otras cosas, porque era capaz de realizar $x$, entonces, también debemos aceptar que quien tolera $x$ en algún momento tenía la capacidad de no tolerar $x .{ }^{17}$ Es decir, sólo es tolerante aquel que tiene la posibilidad de no serlo.

Ahora bien, todos los casos que hasta ahora he ejemplificado me parecen claros. Sin embargo, aún me cuesta trabajo creer que todas las situaciones de competencia queden tan tajantemente reguladas como las antes vistas. Sospecho que al menos hay situaciones en las que la superioridad de un agente frente a otro es tan sólo temporal o pasajera y que está ligada a las circunstancias o contextos en los que se encuentren los agentes. Ésta sería la segunda posibilidad que mencio-

${ }^{17}$ Cfr. E. Rivera López, Presupuestos morales del liberalismo, pp. 153-154. 
né páginas antes. Sería un tipo de superioridad basada en "roles" de tipo social o moral. Este tipo de situaciones, basadas en una especie de "convencionalismos sociales", desdibujan los casos de tolerancia entre iguales que, entre otros, Ernesto Garzón considera posibles.

Afirmar esto se debe a que existe la posibilidad de que personas iguales (con la misma calidad jerárquica) se encuentren en circunstancias que las pongan en una situación de desigualdad; es decir, yo puedo tener un estatus igual (jurídico, moral, social, no importa a cuál nos refiramos ahora) frente a mi amigo; de hecho, tengo el mismo estatus que él en todos los aspectos. Sin embargo, en el caso de que yo sea un fumador empedernido y él no, no es lo mismo encontrarme en su casa que encontrarnos en la mía. No es lo mismo encontrarnos en su oficina que encontrarnos en un parque público, en el pasillo de la universidad o en un restaurante. No resulta lo mismo tratarnos en una reunión de amigos, que tratarnos cuando él preside un comité académico. Circunstancias de esta clase cambian nuestra percepción del estatus de superioridad-inferioridad y, por lo tanto, cambia también el rol de sujeto-objeto de tolerancia.

El trasfondo de esta idea es el make up psicológico que todos los individuos usamos en distintas situaciones; en nuestros roles como padres, como amigos, como profesionales, como compañeros, etc. Esta clase de situaciones logra cambiar nuestra percepción y, con ella, cambia también nuestra impresión sobre el rango de supra-subordinación. Imaginemos una persona que sinceramente acepta que su padre tiene cierta superioridad jerárquica frente a él. En muchas ocasiones, su padre actúa de una forma que él francamente desaprueba; sin embargo, dada la superioridad jerárquica que él le reconoce a su padre no diría que lo tolera, sino, posiblemente, que lo soporta o lo padece. Empero, si una de las acciones cometidas por su padre llegara a afectar a su hijo (es decir, al nieto de su padre), la situación podría cambiar y es probable que él llegue a no tolerarla.

$\mathrm{Si}$ aceptamos estas variables sobre las relaciones de supra-subordinación, me parece que entonces deja de cobrar mucho sentido lo que significa "competencia" en esa segunda forma que decía podría entenderla Garzón; es decir, una situación en la cual dos personas entran en conflicto, y de la cual se dice que una de ellas tiene competencia o superioridad sobre la otra para resolver o decidir la situación, pero, al mismo tiempo, se asegura que no existe una relación supra-subordinación definida. Sea como fuere, lo que hay que notar aquí es que también deja de cobrar sentido hablar de una tolerancia entre iguales. Al menos, desde el punto de vista de la tolerancia privada. 
Si me dejo guiar por las posturas de Nicholson y Raphael, puedo decir que el caso de competencia entre M.A. y Garzón se podría resolver acudiendo a la competencia o la autorización que le otorga a M.A. la justificación de su juicio: "que el tabaco perjudica seriamente la salud. Tanto la salud de quien lo fuma, como la salud de quienes están cerca del fumador."

De ser esto correcto - que del reclamo se deriva una especie de facultad (moral) para detener o frenar los actos de terceros-, entonces, podemos sostener sin el menor empacho que Garzón se encuentra en un estado de subordinación, aun cuando haya leído el libro No Smoking de Robert E. Gooding y, no obstante su lectura, no tenga la menor intención de incluir el fumar dentro del catálogo de actos prohibidos. $^{18}$

Aunque también, quizá, hay otras posibilidades: que M.A., en realidad, no tolera el humo de Garzón, sino que lo soporta o lo padece; en tal caso, Garzón estaría en una posición superior a la de M.A., posición que puede estar fundada en convencionalismos de distinto tipo, como la edad, la posición académica, el respeto que le inspira, o que se encuentren en su despacho o en su casa.

De cualquier manera, la idea que trato de expresar aquí es que ni M.A. ni Garzón se encuentran en una situación de igualdad. Para el caso, podríamos decir que: 1) o M.A. tiene la competencia o la facultad moral que le otorga la justificación de su juicio para impedirle a Garzón que lo dañe físicamente, o 2) que por distintas circunstancias, en realidad él no tolera el humo de Garzón, sino que lo padece o lo soporta. En cualquier caso, repito, Garzón y M.A. estarían en una posición jerárquicamente diferente.

Pero las cosas no terminan aquí. Me parece que Garzón aplica esta distinción con una intención más. Vimos un caso de "tolerancia horizontal privada", pero también hay, según Garzón, casos de "tolerancia horizontal pública". Esta relación de variables le da pie para introducir otra clasificación más: la de una relación triádica simétrica o asimétrica.

La relación triádica consiste en lo siguiente: Garzón entiende la estructura formal de la tolerancia como una relación representada por una persona $a$ que tolera $(T)$ un acto $X$ de otra persona $b$, que es la destinataria de $T$ en una circunstancia específica $c$.

Esta relación puede simplificarse con la fórmula que él usa:

$$
\operatorname{aTbXc} t_{1} \ldots t_{n}
$$

${ }^{18}$ E. Garzón, op. cit., p. 186.

Diánoia, vol. LV, no. 64 (mayo 2010). 
Si la tolerancia es una propiedad disposicional, como afirma Garzón que lo es, tendrá necesariamente —es decir, por definición- que manifestarse en reiteradas ocasiones y circunstancias; esas ocasiones están representadas en esta fórmula por los tiempos $t_{1} \ldots t_{n}$, y como anoté antes, las circunstancias lo están por el símbolo modal $c$.

Ahora bien, respecto de la relación triádica, Garzón sostiene lo siguiente: "en el caso de la tolerancia vertical, la relación triádica es siempre asimétrica; en el de la tolerancia horizontal, puede darse tanto una relación asimétrica como una simétrica". ${ }^{19}$ Por lo tanto, se puede decir que negar la posibilidad de una tolerancia horizontal significa, a su vez, negar una relación triádica simétrica. Pongamos esta situación en claro. La idea que tengo en mente es que la relación de simetría es propia de lo que Garzón llama "tolerancia horizontal" (pues en la tolerancia vertical, por definición, no puede existir una simetría en la relación triádica), entonces, si negamos la existencia de una "tolerancia horizontal pública" negamos la simetría en la relación triádica. Hacer esto puede parecer baladí; sin embargo, sospecho que de hacerlo podemos resaltar alguna vulnerabilidad en la propuesta conceptual de Ernesto Garzón y, sobre todo, negar la posibilidad de una tolerancia entre iguales.

Ahora bien, como referí antes, puede llegar a sostenerse, como Garzón lo hace, que en los casos de tolerancia horizontal privada la simetría sea posible pero no necesaria. Es decir, no siempre hay una conjunción de relaciones triádicas:

$$
a T b X c t_{1} \ldots t_{n} \& b T a Z c t_{1} \ldots t_{n}
$$

Para Garzón, el caso que venimos examinando podría desempeñar un papel tanto simétrico como asimétrico; M.A. puede tolerar y no esperar ser tolerado por Garzón (asimétrica), o tolerar y esperar ser tolerado (simétrica). Sin embargo, como traté de argumentar antes, no creo que éste sea un caso de tolerancia horizontal; por lo tanto, mi postura —según la definición de Garzón — niega la posibilidad de una simetría en la relación triádica.

Pero supongamos por un momento que existe la posibilidad de que M.A. tolera el humo de Garzón y que Garzón, a su vez, tolera algún otro acto de M.A. Esta supuesta simetría, que se puede llegar a dar, no implica necesariamente que se encuentren en una situación de igualdad. En algunos casos, como lo he tratado de argumentar, la situación suprasubordinación varía dependiendo de muchos factores, como podrían

$$
{ }^{19} \text { Ibid., p. } 186 .
$$


ser el rol que una de las partes esté desempeñando, el lugar donde se encuentre, la calidad del juicio moral que ha sido lesionado, etcétera.

Ya analizados algunos puntos relacionados con casos de tolerancia privada, ahora dirijamos la mirada hacia los casos de tolerancia horizontal pública, los que, según Garzón, siempre son simétricos. ${ }^{20}$ Esto se debe a que en estos casos es indispensable la reciprocidad en la tolerancia. ${ }^{21}$ Para Garzón, un buen ejemplo de esta clase de tolerancia (entre iguales) es el contrato social hipotéticamente supuesto como remedio para salir del estado de naturaleza. ${ }^{22}$ Concretamente, según Garzón, "aquí, los actos tolerados son los mismos y las circunstancias también”. ${ }^{23}$

De manera que su representación simbólica se formula así:

$$
(a, b \in C)(a T b X c \supset b T a X c)
$$

Antes dije que, bajo el marco conceptual de Garzón, negar la calidad horizontal de la tolerancia significaría negar, al mismo tiempo, la posibilidad de hablar de una simetría en la relación triádica (es decir, de hablar de una tolerancia entre iguales). Creo haber hecho esto con la tolerancia horizontal privada expuesta con el caso de M.A. Si en lo que sigue logro argumentar con éxito que el pacto social que nos ayuda a salir de un estado de anarquía no implica un acto de tolerancia (ni recíproca entre iguales — la que sería simétrica horizontal—, ni una tolerancia simpliciter —es decir, asimétrica vertical-), sino que, en todo caso, se trata más bien de un acuerdo de voluntades entre individuos que se encuentran en las mismas circunstancias, estaré en posición de negar la simetría de la relación triádica y, por lo tanto, su horizontalidad. De lograr esto, como dije anteriormente, me parece que estaré en condiciones de no otorgarle ninguna importancia conceptual a la dicotomía "privada-pública" de la que nos habla Garzón, entre otros.

${ }^{20}$ Ibid., p. 187.

${ }^{21}$ Ibid.; las cursivas son mías.

${ }^{22}$ Hágase hincapié en que uno de los puntos que autores como Rawls han criticado del contractualismo clásico à la Hobbes es que los participantes en el contrato social, precisamente, no se encontraban en una situación de completa igualdad y que, por lo tanto, el contrato no podría ser considerado legítimo o que partiera de un punto de vista moral. Para un análisis del argumento de Rawls, véase el libro de Roberto Gargarella, Las teorías de la justicia después de Rawls. Un breve manual de filosofía política, pp. 33-34.

${ }^{23}$ E. Garzón, op. cit., p. 187.

Diánoia, vol. LV, no. 64 (mayo 2010). 
Ahora bien, no creo que importe demasiado bajo qué versión del contrato social nos coloquemos, ya sea de corte hobbesiano o lockeano. ${ }^{24}$ En ambos casos, me parece, no cabe hablar de una relación de tolerancia recíproca entre los individuos que lo suscriben. Sin embargo, mi argumento lo centraré en la teoría hobbesiana del contrato social.

Ciertamente, en la teoría del "estado de naturaleza" se define éste como un estado de suma igualdad entre los hombres. Pero ahora recordemos que, para un autor como Hobbes, esta situación de suma igualdad, de igualdad de facto, es una de las condiciones principales que generan la desconfianza entre los individuos. Esta condición en la obra de Hobbes, junto con la escasez de bienes, crea la supuesta "guerra de todos contra todos". ${ }^{25}$

Hobbes incluso diría que de la suma de estos elementos se derivan las tres causas principales de disensión del hombre; es decir: la competencia, la desconfianza y la gloria. ${ }^{26}$ No vayamos más lejos. El estado de naturaleza es la supuesta situación en la que los hombres no pueden actuar de acuerdo con los dictados de la recta razón por la inexistencia de un poder tan irresistiblemente fuerte que garantice a todos los individuos (que deseen actuar de esa forma) que sus contrapartes actuarán de la misma manera.

Los dictados de la razón que refiero, que Hobbes plasma en el Leviatán en una veintena de leyes naturales, según la interpretación de varios filósofos - por ejemplo, Norberto Bobbio-, obligan solamente in foro interno. Esto es, que los individuos deben estar convencidos de que obedecer el dictado de estas leyes condicionadas es la única manera de lograr la paz común; es decir, los individuos están convencidos de que si obedecen estas leyes podrán, finalmente, garantizar su primum bonum: la vida. ${ }^{27}$

Sin embargo, la aceptación interna de dichas leyes no es suficiente; se tiene que dar un paso más. Un paso que nos ayude a abandonar el

${ }^{24}$ Como hago notar, soy consciente de las marcadas diferencias que hay entre las teorías del contrato social de corte hobbesiano, lockeano o rousseauniano; no obstante, considero que el argumento que voy a presentar vale para las tres indistintamente. Aquí, baso mi argumento en la teoría de Thomas Hobbes, porque es la que Garzón cita alguna vez en su trabajo.

${ }^{25}$ Th. Hobbes, Leviatán, vol. I, p. 107.

${ }^{26}$ Recordemos que Hobbes dice: "en la naturaleza del hombre, encontramos tres causas principales de disensión. La primera es la competencia; en segundo lugar, la desconfianza; y en tercer lugar, la gloria. La primera hace que los hombres invadan el terreno de otros para adquirir ganancia; la segunda, para lograr seguridad; y la tercera, para adquirir reputación" (Th. Hobbes, op. cit., p. 107).

${ }^{27}$ N. Bobbio, Thomas Hobbes, pp. 48-49.

Diánoia, vol. LV, no. 64 (mayo 2010). 
estado de naturaleza y, así, arribar al deseado estado civil. Este paso, según Hobbes, sólo se puede dar por la vía del consenso: principio máximo que legitima la sociedad política, según Hobbes.

Bajo este prisma, me queda claro que no se trata de actos de tolerancia. Se trata de un acuerdo de voluntades, de un contrato; de una aceptación voluntaria entre las partes para deshacerse, efectivamente, de una situación insoportable. Pero el contrato mismo - el acuerdo de voluntades mismo- no es un acto de tolerancia recíproca. Es un "acuerdo" entre individuos para superar un estado de anarquía considerado por ellos como un mal en sí mismo.

Para el caso, recordemos una nota que me parece sumamente significativa en este momento: tolerar no significa aceptar. Tolerar significa menguar o detener, por un lapso determinado de tiempo y por determinadas razones, la fuerza normativa de una convicción que se considera relevante y que ha sido lesionada. Esto no es precisamente lo mismo que aceptar, lo cual sería, en pocas palabras, deshacerse de la convicción lesionada; es decir, aceptar sería reconocer que tal o cual prohibición o permisión que teníamos contempladas para determinadas circunstancias estaban mal; que estaban mal desde un punto de vista moral o que estaban mal desde un punto de vista de coherencia intrasistémica, pero que, bajo cualquier punto de vista, era preciso deshacernos de ella.

Decir que el contrato social es un acto de tolerancia es decir que, después de todo, tras su hipotética aceptación, en el momento en que lo deseemos podemos echar a andar de nuevo la prohibición o la permisión que habíamos "menguado" o "detenido" temporalmente. Sólo hay dos salidas para dejar de tolerar: ya sea frenando o deteniendo aquel acto que desaprobamos, o deshaciéndonos de la prohibición; es decir, actuar o aceptar. Situación que en este contexto, me parece, no es el caso. No lo es principalmente porque, como va la trama del paso de un estado de naturaleza a una sociedad civil, en ésta ya se encuentra un poder tan irresistiblemente fuerte, que no me permite regresar a las mismas circunstancias en las que me encontraba antes. Precisamente de eso trata el contrato supuestamente firmado: de aceptar las reglas (los castigos que las siguen) y los derechos que en el juego social se me imponen y se me reconocen con una nota de irrevocabilidad. ${ }^{28}$ Es un paso duradero y estable a un estadio social de mutuo respeto y aceptación.

${ }^{28}$ Cfr. N. Bobbio, op. cit., p. 52. Según Bobbio, la irrevocabilidad es uno de los tres atributos fundamentales de la doctrina hobbesiana del Estado. Los otros dos son: el carácter absoluto y la indivisibilidad.

Diánoia, vol. LV, no. 64 (mayo 2010). 
Me parece que no estoy solo en esto. Javier de Lucas, por ejemplo, en su trabajo "¿Para dejar de hablar de la tolerancia?" afirma que:

las situaciones de "tolerancia horizontal" [que argumenta Garzón], en el ámbito público son o bien irrelevantes o bien inexistentes [...]; lo que Garzón describe como tales ("el contrato social hipotéticamente supuesto como remedio a los costos de la anarquía") [...] no son ejemplos de tolerancia horizontal, sino expresiones de los términos en los que se enmarca el consenso político fundacional, esto es, de derechos: quien pacta en términos de igualdad no pretende tolerancia, sino reconocimiento [de sus] derechos. ${ }^{29}$

Si todo esto es correcto, diría entonces que la horizontalidad en la tolerancia no es tal. Y como ya lo había expresado páginas atrás, esta situación implica que hablar de una simetría en la relación triádica de la tolerancia podría tomarse como un error conceptual o teórico.

$\mathrm{Al}$ inicio de este trabajo me pregunté si la distinción entre tolerancia pública y privada tenía alguna relevancia conceptual. Frente a ello, recuérdese que anoté que Garzón sostenía esta distinción con la otra diferencia entre tolerancia horizontal y vertical, y con la necesidad de que la relación triádica fuera simétrica en el caso de la tolerancia horizontal pública. Sin embargo, como he argumentado a lo largo de estas páginas, ni la simetría de la relación triádica ni la horizontalidad de la tolerancia me parecen distinciones plausibles. Por lo tanto, sospecho que la relevancia conceptual de la dicotomía "pública-privada" tampoco lo es.*

\section{BIBLIOGRAFÍA}

Alexy, R., Teoría de los derechos fundamentales, trad. Ernesto Garzón Valdés, Centro de Estudios Constitucionales, Madrid, 1993.

Atienza, M., "La filosofía moral de Ernesto Garzón Valdés", en Ernesto Garzón Valdés, Derecho, ética y política, Centro de Estudios Constitucionales, Madrid, 1993, pp. 21-32.

Bobbio, N., Thomas Hobbes, trad. Manuel Escrivá de Romaní, Fondo de Cultura Económica, México, 1995.

${ }^{29}$ J. de Lucas, “¿Para dejar de hablar de tolerancia?”, específicamente la p. 121.

*Agradezco los comentarios que hicieron los árbitros de Diánoia a la versión preliminar de este trabajo. Aprovecho la oportunidad para agradecer también a Rodolfo Vázquez la paciencia que ha tenido para platicar y discutir muchos de estos temas conmigo; estas conversaciones, sin duda, me han ayudado a comprender mejor algunos problemas que giran alrededor de la tolerancia. 
Cohen, A.J., "What Toleration Is", Ethics, vol. 115, no. 1, 2004, pp. 68-95.

De Lucas, J., “iPara dejar de hablar de la tolerancia?”, Doxa. Cuadernos de filosofía del derecho, no. 11, 1992, pp. 117-126.

Gargarella, R., Las teorías de la justicia después de Rawls. Un breve manual de filosofía política, Paidós, Barcelona, 1999.

Garzón Valdés, E., "'No pongas tus sucias manos sobre Mozart'. Algunas consideraciones sobre el concepto de tolerancia", en Instituciones suicidas. Estudios de ética y política, Paidós/Facultad de Filosofía y Letras-UnAM, México, 2000, pp. 181-198.

González de la Vega, R., "Desacuerdos en el concepto de tolerancia", Jurídica. Anuario del Departamento de Derecho de la Universidad Iberoamericana, no. 37, Universidad Iberoamericana, México, 2007, pp. 373-394.

Heyd, D. (comp.), Toleration. An Elusive Virtue, Princeton University Press, Princeton, 1996.

Hobbes, Th., Leviatán, vol. I, trad. Carlos Mellizo, Alianza, Madrid, 1992 (Grandes Obras del Pensamiento).

Horton, J., "Toleration as a Virtue", en D. Heyd (comp.), Toleration. An Elusive Virtue, pp. 28-43.

Mendus, S. (comp.), Justifying Toleration. Conceptual and Historical Perspectives, Cambridge University Press, Nueva York, 1988.

Nicholson, P., "Toleration as a Moral Ideal", en J. Horton y S. Mendus (comps.), Aspects of Toleration, Methuen, Londres/Nueva York, 1985, pp. 158-173.

Raphael, D.D., "The Intolerable", en S. Mendus (comp.), Justifying Toleration. Conceptual and Historical Perspectives, Cambridge University Press, Nueva York, 1988, pp. 137-153.

Rivera López, Eduardo, Presupuestos morales del liberalismo, Centro de Estudios Constitucionales, Madrid, 1997.

Scanlon, M., "The Difficulty of Tolerance", en D. Heyd (comp.), Toleration. An Elusive Virtue, pp. 226-239.

Schmit, A., "Las circunstancias de la tolerancia", Doxa. Cuadernos de Filosofía del Derecho, no. 11, 1992, pp. 71-85.

Vázquez, R., Liberalismo, Estado de derecho y minorías, Paidós/Facultad de Filosofía y Letras-UnAm, México, 2001.

_- Entre la libertad y la igualdad. Introducción a la filosofía del derecho, Trotta, Madrid, 2006.

Vernon, R. y S. LaSelva, "Justifying Tolerance", Canadian Journal of Political Science, vol. 17, no. 1, 1984, pp. 3-23.

Vicent, M., No pongas tus sucias manos sobre Mozart, Debate, Madrid, 1983.

Warnock, M., "The Limits of Toleration", en S. Mendus y D. Edwards (comps.), On Toleration, Clarendon Press, Oxford, 1987, pp. 123-139.

Recibido el 18 de junio de 2008, aceptado el 14 de octubre de 2009. 